\title{
OPTIMIZATION OF SATELLITE COMBINATION IN KINEMATIC POSITIONING MODE WITH THE AID OF GENETIC ALGORITHM
}

\author{
Panithan Srinuandee ${ }^{1}$, Chalermchon Satirapod ${ }^{1}$, Clement Ogaja ${ }^{2}$ and Hung-Kyu Lee ${ }^{3}$ \\ ${ }^{1}$ Department of Survey Engineering, Faculty of Engineering, Chulalongkorn University, \\ Thailand \\ ${ }^{2}$ Department of Civil \& Geomatics Engineering, California State University, Fresno, California, \\ U.S.A. \\ ${ }^{3}$ Department of Civil Engineering, Changwon National University, Rep. of Korea \\ E-mail: panithan@up.co.th, Chalermchon.s@chula.ac.th (corresponding author), \\ cogaja@csufresno.edu and hkyulee@,changwon.ac.kr
}

\begin{abstract}
The basis of high precision relative positioning is the use of carrier phase measurements. Data differencing techniques are one of the keys to achieving high precision positioning results as they can significantly reduce a variety of errors or biases in the observations and models. Since GPS observations are usually contaminated by many errors such as the atmospheric biases, the receiver clock bias, the satellite clock bias, and so on, it is impossible to model all systematic errors in the functional model. Although the data differencing techniques are widely used for constructing the functional model, some un-modeled systematic biases still remain in the GPS observations following such differencing. Another key to achieving high precision positioning results is to fix the initial carrier phase ambiguities to their theoretical integer values. To obtain a high percentage of successful ambiguity-fixed rates, noisy GPS satellites have to be identified and removed from the data processing step. This paper introduces a new method using genetic algorithm (GA) to optimize the best combination of GPS satellites which yields the highest number of successful ambiguity-fixed solutions in kinematic positioning mode. The results indicate that the use of GA can produce higher number of ambiguity-fixed solutions than the standard data processing technique.
\end{abstract}

Keywords: Global Positioning System, Kinematic Positioning, Genetic Algorithm

\section{INTRODUCTION}

The relative kinematic positioning mode is widely used in many applications since it can provide high precision positioning results especially for moving platforms. There are various techniques of relative kinematic positioning, such as, RTK (Real-Time Kinematic), NRTK (Network RealTime Kinematic), and Post-Processing Kinematic (PPK). Each of these techniques has its own pros and cons. For example, the users can obtain instantaneous precise positioning results in the field using either RTK or NRTK mode while the users of the PPK mode have to wait until the 
data are downloaded and processed in the computer. However, the PPK mode can provide more reliable positioning results and it does not require a communication link between base and roving receivers. In practice, the PPK technique is still widely used in many applications (e.g., vessel tracking, aircraft tracking, monitoring of high-rise buildings and dams). Generally, high quality PPK positioning is possible in good observing environments, but less favorable environments can yield unreliable positioning results. In the latter case, the users have to edit or filter out noisy data at the processing step. The data filtering process can be considered as a trial-and-error process. The user has to re-process over and over until satisfactory ambiguity-fixed solutions are obtained. Often, the data filtering is a time-consuming process that requires the skills of an experienced user.

One of the ways to avoid the above-mentioned problem is to identify the best satellite combination. This can be done with the aid of an optimization technique known as the genetic algorithm (GA). The GA is a method based on a natural evaluation and it aims to search for a global optimum solution from the entire search space. The GA has been successfully applied in many practical applications (Allen and Karjalainen, 1999; Kim et al., 2005; Stomeo et al., 2006; Ran et al., 2010) and has recently been introduced to the field of GPS data processing (Xu et al., 2002; Wu et al., 2007; Mosavi and Divband, 2010; Liu et al., 2010). In this paper, the GA is proposed as a suitable method to optimize the combination of GPS satellites in kinematic positioning. It is employed to search for the best satellite combination which yields the highest number of ambiguity-fixed solutions in kinematic positioning. The proposed method is demonstrated in a PPK mode using sample data from a dedicated network of GPS receivers in Thailand. The paper is structured as follows. Firstly, the concept of the GA is briefly described. Then, the data processing methodology with the PPK mode is explained. Next, discussion and analysis of data are presented. Lastly, some concluding remarks are made.

\section{GENETIC ALGORITHM}

Genetic Algorithm (GA) is the global optimum search algorithm that mimics the natural evolution known as Darwinian Evolution. Because of its advantages (simple, supports parallel computation, immune to noise), it has been extensively used in various fields of study and applications (Holland, 1975). The GA is based on an iterative procedure, an "evolutionary" process, in which it tries to extract the best solution out of a search space without any apriori knowledge or mathematical model related to the problem. It consists of a constant of population size of "chromosomes." Each chromosome is a combination of genes which comprises all possible solutions to a specific problem. There are several manners to represent the gene such as binary, real number, character and so on. However, the binary is generally used.

The GA procedure can be summarized as follows. An initial population of individual chromosome is randomly created. For each evolutionary step also known as generation, each chromosome will be assigned a fitness score based on its ability of solving a problem. The fitness score can be obtained by decoding an individual chromosome and passing through a fitness function. Subsequently, a criterion will be evaluated. If the criterion is not met, then a new set of population for the next generation will be created. Typically, the criterion can be either a number of generations or closeness to the solution. To establish a new set of population, the chromosome will be selected from the entire populations based on a "genetical" selection method. Currently, there are many genetical selection methods being used but the fitness-proportionate selection method is normally used. The fitness-proportionate selection method will select an individual 
chromosome based on its fitness score. Thus, a high-score fitness chromosome tends to have better chance to reproduce a new offspring, while a low-score fitness chromosome is more likely to disappear.

However, the implementation of the fitness-proportionate selection method alone is not sufficient since the GA may not lead to a converged solution. Therefore, genetically-based operators, known as a crossover and mutation, are needed to create new point in the search space. If the probability between two selected parents is lower than a crossover rate, the crossover will be performed by swapping their genes at randomly selected point along the length of the chromosome. Figure 1 illustrates the crossover process. The mutation is the method to add change to each gene in chromosome of the offspring with very small probability (called mutation rate), the mutation process is illustrated in Figure 2. The change is required since it yields diversity to the population and sometime it produces an outstanding advantage among the group. On the other hand, the mutation process aims to prevent the diversity of the solution to the local optima. Figure 3 shows the operating procedure of the standard GA (Melanie, 1999; Buckland, 2002).

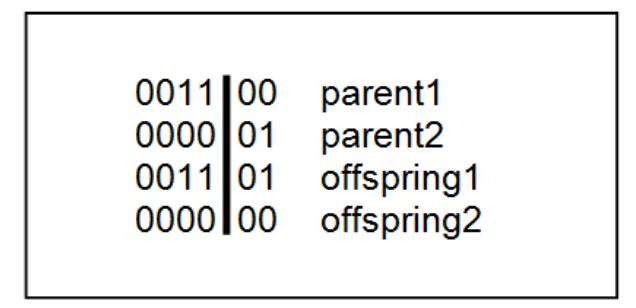

Fig. 1. The crossover process performed at the crossover point as shown in black line

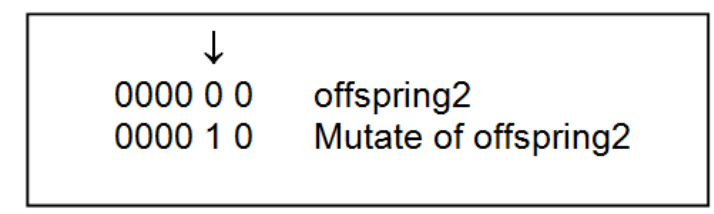

Fig. 2. The mutation process performed at the black arrow

The GA has been introduced in the field of GPS data processing by many researchers. Examples include use of GA in i) GPS attitude determination (Xu et al., 2002), ii) coordinate transformation (Wu et al., 2007), iii) enhancing the search of optimum success rate in GPS carrier phase relative positioning (Liu et al., 2007), iv) approximating GDOP (Mosavi and Divband, 2010), and v) aiding a single epoch ambiguity resolution (Liu et al., 2010). 


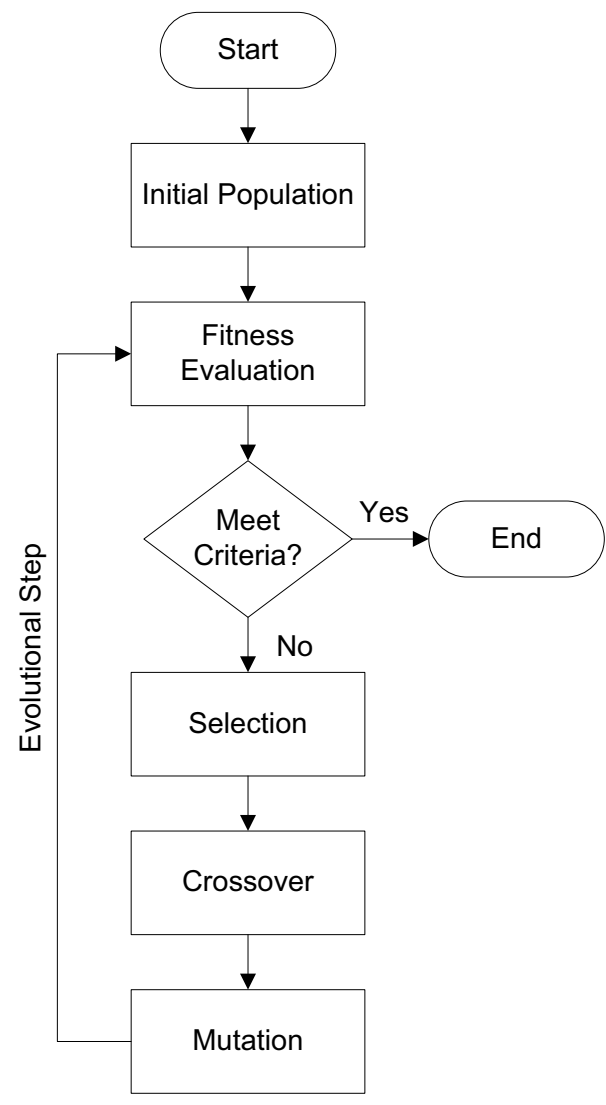

Fig 3. Operating procedure of standard Genetic Algorithm

\section{POST-PROCESSING KINEMATIC MODE WITH THE AID OF GA}

It is relatively straightforward to apply the GA with the PPK mode. However, the success of the application depends mostly on the flexibility of the data processing engine (e.g., in terms of configuration for interaction with an external program). The interaction between the GA and the data processing engine is in the sense that the GA will firstly search for the best solution for each generation and subsequently the data processing engine will use the best solution as its configuration to carry on a standard processing step. The obtained result will then be evaluated by an objective function to decide which member in the population should be survived in the next evolution step. This workflow will continue until the criterion is met. Figure 4 presents a workflow of the proposed PPK with the aid of GA. In order to demonstrate the performance of the proposed procedure, the open-source RTKLib GNSS software package version 2.4.1 (Takasu, 2011) was selected since the RTKLib software can be easily modified to work with an external program. The GA configurations used in this study are shown in Table 1. From Table 1, it is important to note that a population type and an objective function used in the GA have to be decided by a user. The details of the population type and the objective function are described in subsequent subsections. 


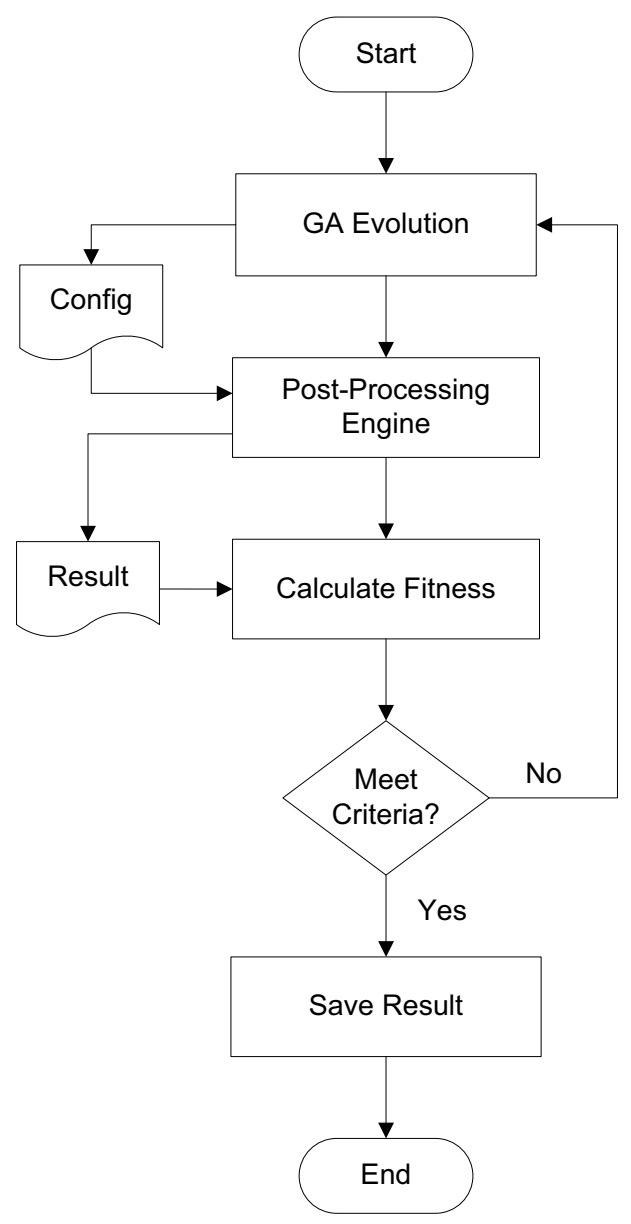

Fig. 4. Operating procedure of optimized Genetic Algorithm with the PPK

Table 1. GA solver configuration within MATLAB

\begin{tabular}{|l|l|}
\hline GA Setting & Value \\
\hline population type & Bit string \\
\hline population size & 20 \\
\hline objective function & $@$ myObjFunc \\
\hline number of generation & 30 \\
\hline stall generation limit & 15 \\
\hline initial population data & {$[1,1,1, \ldots, 1]_{1 \times 32}$} \\
\hline scaling function & Rank \\
\hline selection function & roulette \\
\hline elite count & 2 \\
\hline crossover fraction & 0.8 \\
\hline mutation function & constraint dependent default \\
\hline crossover function & two point \\
\hline
\end{tabular}




\subsection{The Population Type}

As mentioned in the previous section, there are several types of population that GA can understand such as binary, real number and so on. To obtain reliable and accurate positioning, the best satellite combination will be searched for by the GA. Thus, all satellites must be encoded into a proper format. In this study, satellites are encoded using the binary format. Therefore, each satellite will be represented by either ' 0 ' (off) or ' 1 ' (on). The satellite vehicle number (SVN) will be represented by a location of bit in the bits string. Examples of encoding and its meaning are shown in Table 2.

Table 2. The Encoding of problem in bits string and its meaning

\begin{tabular}{|l|l|}
\hline Bits String & Meaning \\
\hline$[1,1,1, \ldots \ldots 1]_{1 \times 32}$ & All satellites are used \\
\hline$[0,1,1, \ldots \ldots 1]_{1 \times 32}$ & Only $\mathrm{SVN}=1$ is excluded \\
\hline$[0,0,0, \ldots \ldots 0]_{1 \times 32}$ & All satellites are excluded \\
\hline
\end{tabular}

\subsection{The Objective Function}

Defining the objective function is the crucial part of the GA. The result obtained from the data processing engine will be used to evaluate the fitness of each individual member in the population. The objective function can be defined as

$$
\text { Fitnesss }=\sum_{i=1}^{n} w_{i} X_{i}
$$

In Equation (1), $n$ is the number of fitness parameters; $X_{i}$ is the fitness parameter; and $w_{i}$ is the weight of the fitness parameter

The number of ambiguity-fixed solutions, the number ambiguity-float solutions, and the number of instantaneous solutions are selected as fitness parameters which will be extracted from the results obtained from the standard data processing step. In addition, the success of the solution can be judged by the number of ambiguity-fixed solutions. In this study, the simple penalty approach (Yeniay, 2005) was employed to reduce the fitness values of ambiguity-float and instantaneous solutions. The weight components for ambiguity-fixed, ambiguity-float, and instantaneous solutions are set to 1,10 and 100 respectively. The interaction between the GA software and the RTKLib software will be controlled by a configuration file in the RTKLib software package. In this study, only the change of satellite combination is allowed while the standard processing parameters remain the sam for all processing rounds.

\section{EXPERIMENTAL RESULTS AND ANALYSES}

\subsection{Data Acquisition}

GPS data used in this experiment were collected using two dual-frequency geodetic receivers with a baseline length (distance between the two receivers) of approximately $17.6 \mathrm{~km}$. The data were collected in static mode on 20 November 2010 using a common observation interval of 5second data rate. Both stations had very good observing environments since they are each set up on top of a building (see Figure 5). For kinematic analysis, the PKKT station is selected as a 
reference station while the DPT9 station is used as a roving station. To validate the performance of the proposed method, the data obtained from the DPT9 station will be treated as kinematic data set.

\subsection{Data Processing}

As a first step, 24-hour long data from the DPT9 station was processed in static mode using the Leica Geomatic Office (LGO) software version 5.0. The resulting coordinates obtained were accurate to within a few millimeters and thus suitable for use as reference or "known coordinates" for later comparison with kinematically processed data.

Kinematic data processing was carried out using the RTKLib software. The 24-hr data set was segmented into 1-hr sessions, and hence, a total of 24 sessions were obtained. Each 1-hr session was processed using both the proposed procedure (with the aid of GA) and the standard procedure (without the aid of GA).

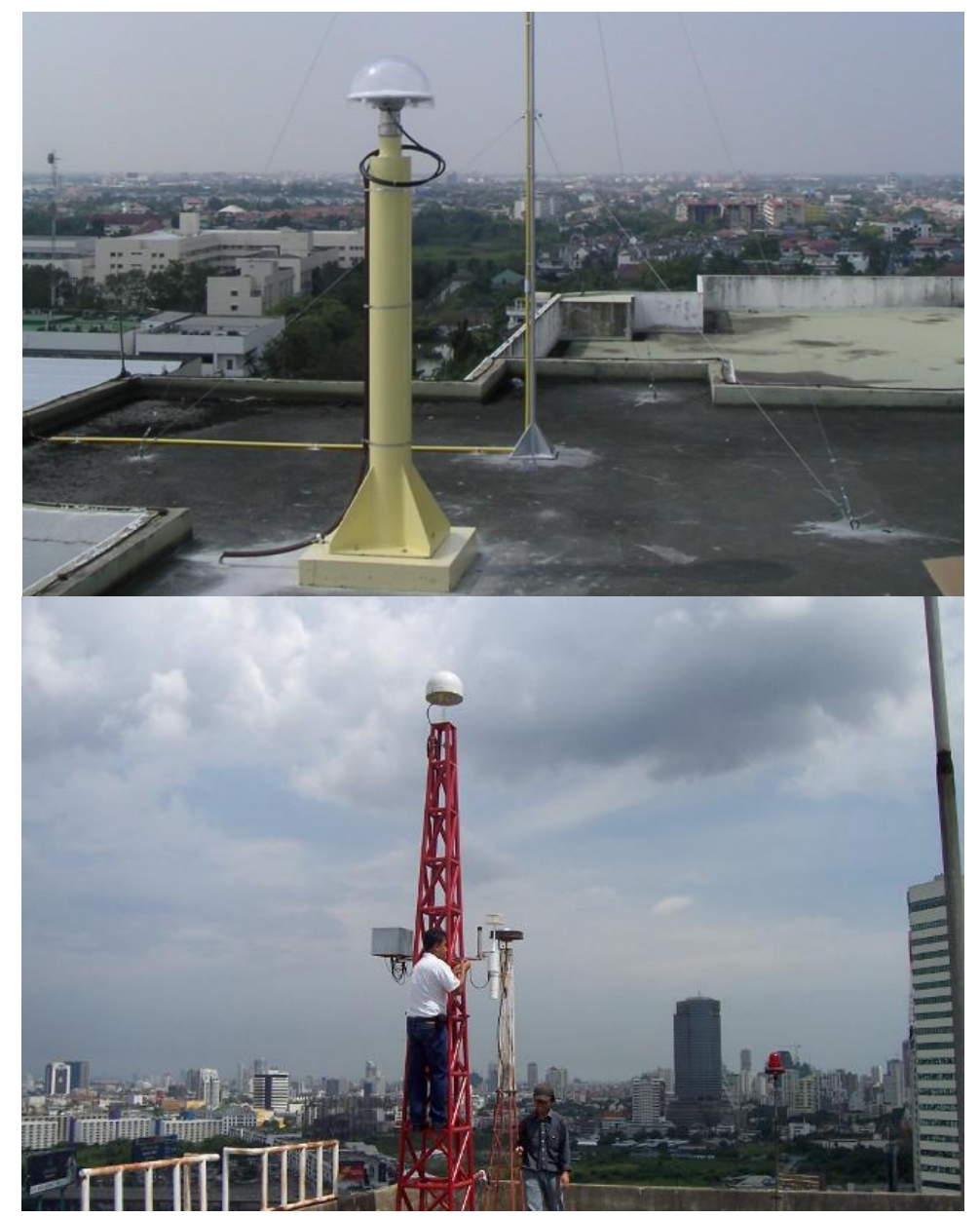

Fig. 5. Top: The PKKT station (base station), Bottom: The DPT9 station (roving station)

The results obtained from the proposed procedure were compared with the results from the standard procedure to determine the levels of improvement in the number of ambiguity-fixed 
solutions. We define four levels of improvement according to the classification shown in Table 3 . Using this classification, the frequency bin of each improvement level was counted and plotted as shown in Figure 6.

Table 3. The classification interval of improvement

\begin{tabular}{|l|l|}
\hline Level & Percentage of improvement \\
\hline No improvement & 0 \\
\hline Low improvement & $0.01-10.00 \%$ \\
\hline Moderate improvement & $10.01-20.00 \%$ \\
\hline High improvement & $>20.01 \%$ \\
\hline
\end{tabular}

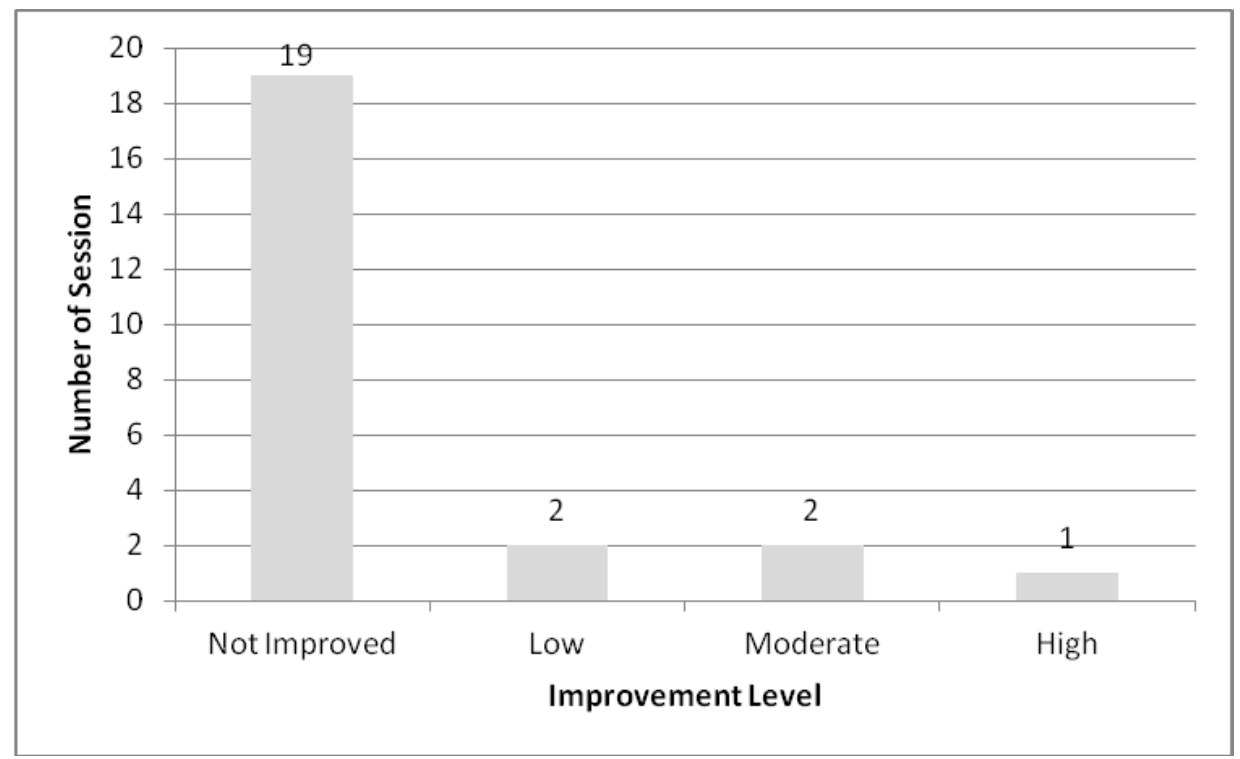

Fig. 6. The improvement classification of results from the proposed procedure

\subsection{Analysis of Results}

For subsequent analyses, assume that the consecutive "1-hr" sessions are alphabetically referred to using the letters A, B, C, D, .., and so forth. Using this scheme, and on the basis of classified results as shown in Figure 6, sessions D, F and Q were selected as representative for "High", "Moderate" and "Low" improvements, respectively. The best satellite combination for each of the sessions D, F and $\mathrm{Q}$ as identified by the GA is shown in Table 4. The respective discrepancies in the three coordinate components $(\mathrm{N}, \mathrm{E}, \mathrm{H}$ ) (solutions compared to known coordinates) for each of these sessions are plotted in Figures 7, 8 and 9. In each subplot, the $\mathrm{x}$ axis represents the solution epoch and the y-axis represents the magnitude of errors in units of meters. Grey line represents the results obtained from the standard PPK while the black line represents the results from the proposed procedure. 
Table 4. The best satellite combination identified by GA

\begin{tabular}{|c|l|l|}
\hline Session & Available Satellites & Best satellite combination \\
\hline D & $2,4,7,8,10,11,17,20,28$ & $4,7,8,11,17,20,28$ \\
\hline F & $2,4,5,8,9,10,12,17,20,27,28$ & $2,4,5,8,9,10,12,20,28$ \\
\hline Q & $12,14,16,18,21,22,24,25,30,31$ & $12,14,16,18,22,24,25,30,31$ \\
\hline
\end{tabular}

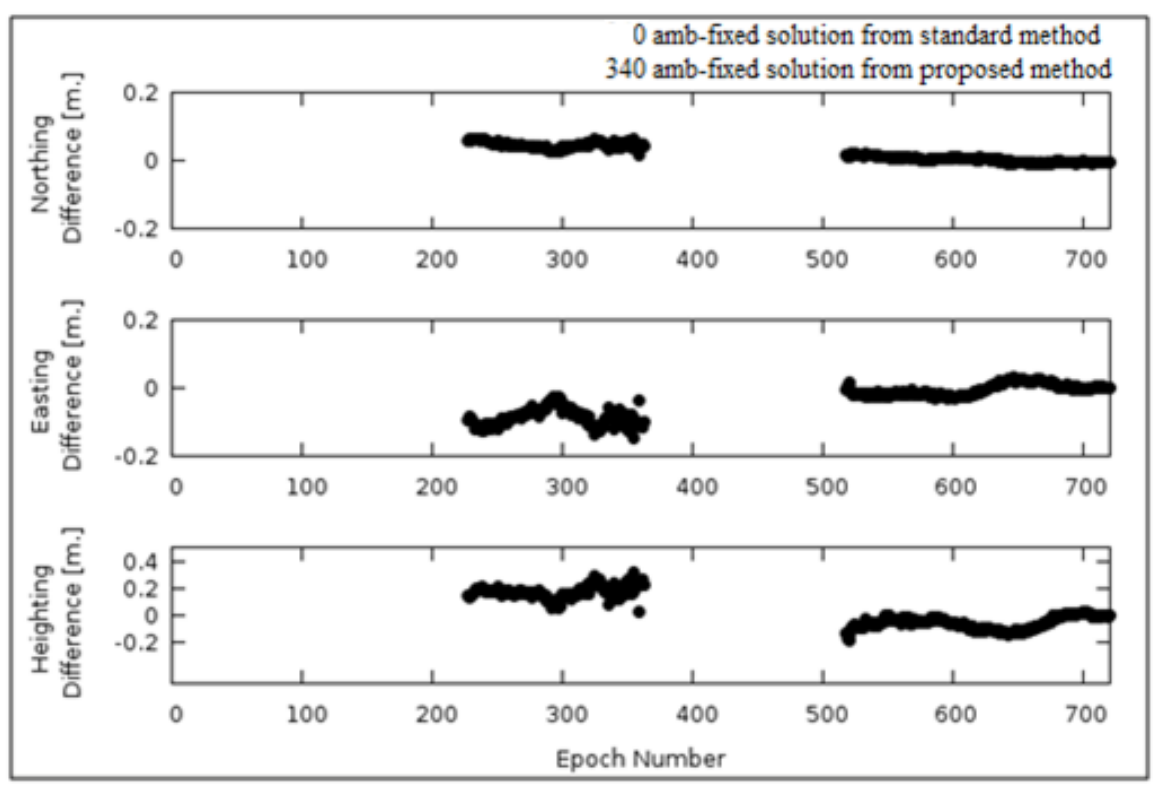

Fig. 7. Differences between the solutions and the known coordinates for session D

(Top: North component; Middle: East component; Bottom: Height component)

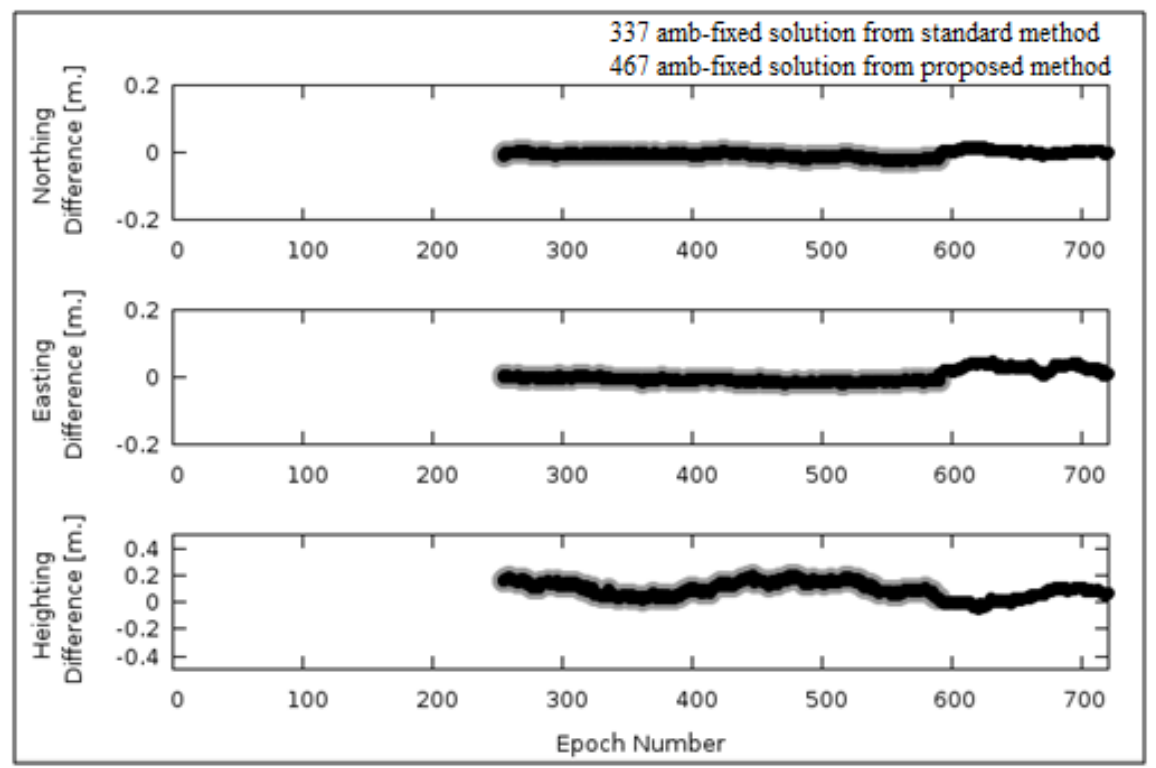

Fig. 8. Differences between the solutions and the known coordinates for session $F$ (Top: North component; Middle: East component; Bottom: Height component) 


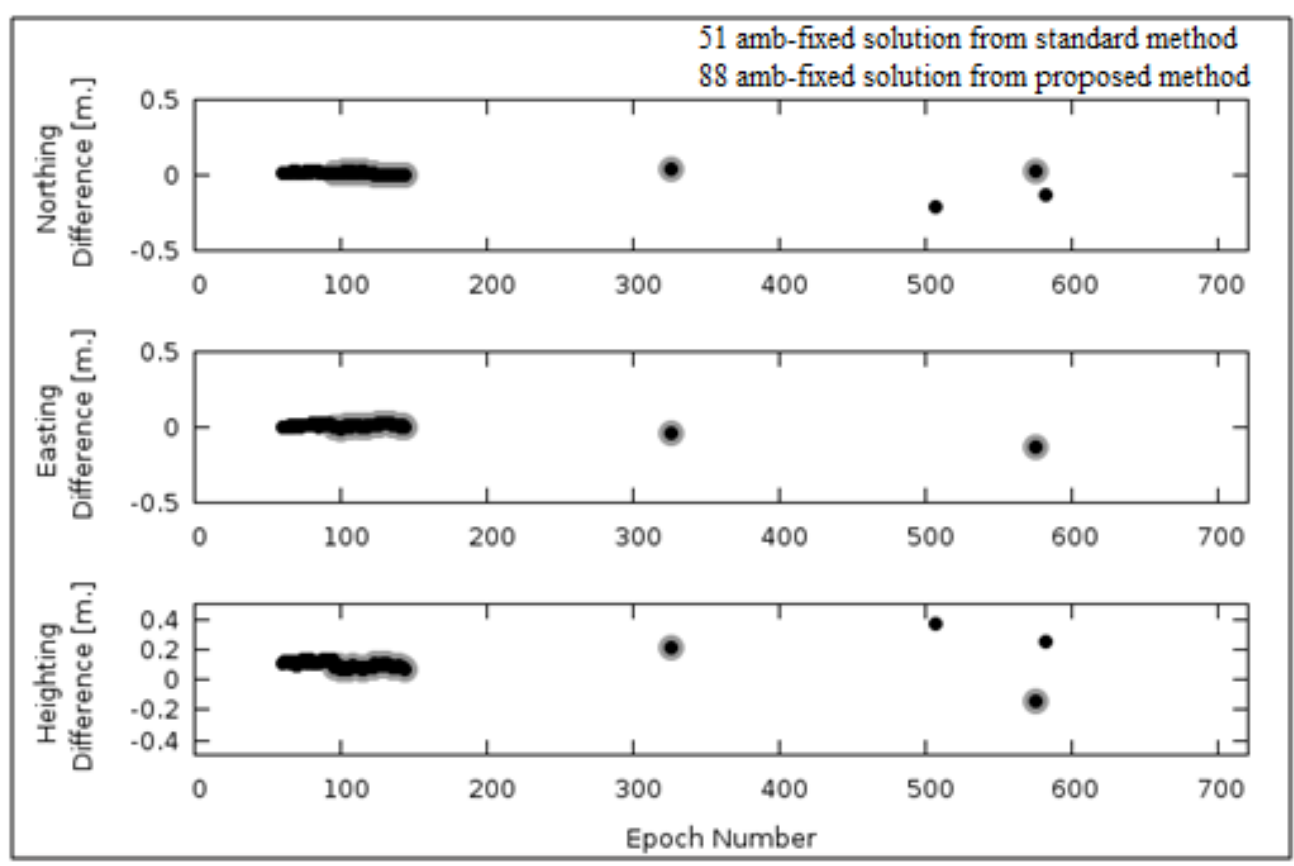

Fig. 9 Differences between the solutions and the known coordinates for session Q (Top: North component; Middle: East component; Bottom: Height component)

It can be seen from Figure 6 that the proposed procedure can improve the number of ambiguityfixed solutions up to 5 sessions out of 24 sessions. With reference to Table 4 and Figures 7-9, it is clearly evident that the use of all available satellites may not always lead to optimal solutions. In some cases, a deletion of specific satellites from the data processing can yield better results. Thus, it can be concluded that the proposed procedure can improve the success rate of ambiguity resolution in the kinematic positioning mode. However, in terms of the positioning accuracy, it can be seen from Figures 7-9 that the positioning results obtained from the proposed procedure are at the same level as the results from the standard PPK. This is due to the fact that the objective function of the GA is designed to only improve the success rate of ambiguity resolution. It is very difficult to set the objective function to improve the positioning accuracy since we do not know the true coordinate for each solution in the real world.

\section{CONCLUDING REMARKS}

In this paper, the concept of genetic algorithm (GA) and its application in GNSS kinematic positioning is presented. The GA has been successfully introduced to the post-processing kinematic mode. The primary goal of the GA is to optimize the selection of best satellite combination in the data processing step. The results obtained from the proposed procedure indicate that it can improve the success rate of ambiguity resolution. Furthermore, it has the potential benefit of being used to replace the manual pre-processing of GPS data. Further investigations are being carried out to enhance the use of the algorithm, including as a possible aid for improving the reliability of positioning results. 


\section{ACKNOWLEDGEMENT}

The authors would like to thank to the Department of Public Works and Town \& Country Planning (DPT), The Department of Land (DOL) and Ultimate Positioning Co., Ltd. for providing the GPS data used in this study.

\section{REFERENCES}

Allen, F., and Karjalainen, R. (1999) Using Genetic Algorithms to Find Technical Trading Rules. Journal of Financial Economics 51, pp. 245-271.

Buckland, M. (2002) AI Technique for Game Programming. The Premier Press Game Development Series, Premier Press.

Holland, J. H. (1975) Adaptation in Natural and Artificial Systems. The University of Michigan Press, Ann Arbor

Kim, S., Ito, K., Yoshihara, D., and Wakisaka, T. (2005) Application of a Genetic Algorithm to the Optimization of Rate Constants in Chemical Kinetic Models for Combustion Simulation of HCCI Engines. JSME International Journal Series B, 48(4), pp.717-724.

Liu, Z., Du, Z., Zou, R. (2007) Application of the Improved Genetic Algorithms With Real Code on GPS Data Processing. International Conference on Natural Computation, pp. 420424.

Liu, Z., Xiong, W., Kang, Z., Zhang, H., Qu, M. (2010) GPS Ambiguity Resolution of Single Epoch Data Using genetic algorithms. International Conference on Natural Computation, pp. 2365-2368.

Melanie, M. (1999) An Introduction to Genetic Algorithms(Complex Adaptive Systems), 5th ed., MIT Press.

Mosavi, M. R., and Divband, M. (2010) Calculation of Geometric Dilution of Precision using Adaptive Filtering Technique based on Evolutionary Algorithms. IEEE International Conference on Electrical and Control Engineering, pp.4842-4845.

Ran, Y., Xiong, G., Li, S., and Ye, L. (2010) Study on deformation prediction of landslide based on genetic algorithm and improved BP neural network. Kybernetes, 39(8), pp.1245-1254.

Stomeo, E., Kalgonova, T., and Lambert, C. (2006) A Novel Genetic Algorithm for Evolvable Hardware. IEEE Congress on Evolutionary Computation, Sheraton Vancouver Wall Centre Hotel, Vancouver, BC, Canada.

Takasu, T. (2011) RTKLIB: An Open Source Program Package for GNSS Positioning. http://www.rtklib.com. Accessed 08 February 2012.

Wu, C., Chou, H., and Su, W. (2007) A Genetic Approach for Coordinate Transformation Test of GPS Positioning. IEEE, Geoscience And Remote Sensing Letters, 4(2), pp.297-301. 
Xu, J., Arslan, T., Wang, Q., and Wan, D. (2002) An EHW Architecture for Real-Time GPS Attitude Determination Based on Parallel Genetic Algorithm. Proceedings of the 2002 NASA/DOD Conference on Evolvable Hardware, VA, USA.

Yeniay, Ö. (2005) Penalty Function Method for Constrained Optimization with Genetic Algorithms. Mathematical and Computational Applications, 10(1), pp. 45-56.

Received: 2012-04-04,

Reviewed: 2012-04-25, by Zhimin Liu,

Accepted: 2012-04-27. 